\title{
Comparative evaluation of the Ifakara tent trap-B, the standardized resting boxes and the human landing catch for sampling malaria vectors and other mosquitoes in urban Dar es Salaam, Tanzania Maggy Sikulu*1, Nicodem J Govella ${ }^{2,3}$, Sheila B Ogoma ${ }^{1}$, John Mpangile ${ }^{2,4}$, Said H Kambi ${ }^{2,4}$, Khadija Kannady ${ }^{4}$ Prosper C Chaki ${ }^{2,3}$, Wolfgang R Mukabana ${ }^{1}$ and Gerry F Killeen ${ }^{2,3,5}$
}

Address: ${ }^{1}$ Department of Zoology, University of Nairobi, PO Box 30197-00100 GPO Nairobi, Kenya, ${ }^{2}$ Ifakara Health Institute, Coordination Office, PO Box 78373, Kiko Avenue, Mikocheni A, Dar es Salaam, United Republic of Tanzania, ${ }^{3}$ Durham University, School of Biological and Biomedical Sciences, South Road, Durham, DH1 3LE, UK, ${ }^{4}$ Dar es Salaam City Council, Ministry of Regional Administration and Local Government, United Republic of Tanzania and ${ }^{5}$ Liverpool School of Tropical Medicine, Vector Group, Pembroke Place, Liverpool, L3 5QA, UK

Email: Maggy Sikulu* - maggysikulu@yahoo.com; Nicodem J Govella - govella@ihi.or.tz; Sheila B Ogoma - ogomasheila@yahoo.com; John Mpangile - jmpangile@yahoo.co.uk; Said H Kambi - kambi_lee@yahoo.co.uk; Khadija Kannady - kadkhadija@yahoo.co.uk; Prosper C Chaki - pchaki@ihi.or.tz; Wolfgang R Mukabana - rmukabana@yahoo.co.uk; Gerry F Killeen - gkilleen@ihi.or.tz

* Corresponding author

Published: 12 August 2009

Malaria Journal 2009, 8:197 doi:10.1186/1475-2875-8-197

This article is available from: http://www.malariajournal.com/content/8/1/197

(c) 2009 Sikulu et al; licensee BioMed Central Ltd.

This is an Open Access article distributed under the terms of the Creative Commons Attribution License (http://creativecommons.org/licenses/by/2.0), which permits unrestricted use, distribution, and reproduction in any medium, provided the original work is properly cited.
Received: 16 January 2009

Accepted: 12 August 2009

\begin{abstract}
Background: Frequent, sensitive and accurate sampling of Anopheles mosquitoes is a prerequisite for effective management of malaria vector control programmes. The most reliable existing means to measure mosquito density is the human landing catch (HLC). However, the HLC technique raises major ethical concerns because of the necessity to expose humans to vectors of malaria and a variety of other pathogens. Furthermore, it is a very arduous undertaking that requires intense supervision, which is severely limiting in terms of affordability and sustainability.

Methods: A community-based, mosquito sampling protocol, using the lfakara tent trap-B (ITT-B) and standardized resting boxes (SRB), was developed and evaluated in terms of the number and sample composition of mosquitoes caught by each, compared to rigorously controlled HLC. Mosquitoes were collected once and three times every week by the HLC and the alternative methods, respectively, in the same time and location.

Results: Overall, the three traps caught 44,848 mosquitoes. The ITT-B, HLC and SRB caught I68, I43 and 46 Anopheles gambiae s.l. as well as 26,3I5, I3,258 and 4,79I Culex species respectively. The ITT-B was three- and five-times cheaper than the HLC per mosquito caught for An. gambiae and $C_{x}$. Species, respectively. Significant correlations between the numbers caught by HLC and ITT-B were observed for both An. gambiae s.I. $(\mathrm{P}<0.00 \mathrm{I})$ and $C_{x}$. species $(P=0.003)$. Correlation between the catches with HLC and SRB were observed for $C_{x}$. species $(P<0.00 I)$ but not An. gambiae s.l. $(P=0.195)$, presumably because of the low density of the latter. Neither ITT$B$ nor SRB exhibited any obvious density dependence for sampling the two species.

Conclusion: SRBs exhibited poor sensitivity for both mosquito taxa and are not recommended in this setting. However, this protocol is affordable and effective for routine use of the ITT-B under programmatic conditions. Nevertheless, it is recommended that the trap and the protocol be evaluated further at full programmatic scales to establish effectiveness under fully representative conditions of routine practice.
\end{abstract}




\section{Background}

Monitoring and evaluation of malaria control interventions and their associated impact on malaria burden is essential for understanding progress, successes and challenges in any malaria control effort [1]. In order to accurately estimate and manage the burden of a disease and measure the trends in malaria transmission intensity, more practical and cost-effective survey instruments and methods are needed to monitor the densities of the adult mosquito populations $[2,3]$.

In urban Dar es Salaam, the Urban Malaria Control Programme (UMCP) relies upon the human landing catch (HLC) for entomological surveys of malaria vectors, transmission intensity and for evaluation of regular larvicide application [4-6]. Nonetheless, the HLC is difficult to supervise, unreliable, expensive, labour-intensive and requires skilled catchers. It is also not representative of true human exposure as it is usually implemented by adult males who remain awake and seated all night. However, the most serious problem arises when human participants are at an increased risk of malaria infection [7]. Many other methods e.g. the CDC light trap and the Mbita bed net trap have been employed and evaluated in urban Dar es Salaam as alternatives to HLC but none has proven to approach adequate sensitivity [8].

Elsewhere, resting boxes have been used to sample mosquitoes, relying on the widely observed phenomenon that they congregate in diurnal resting places which are dark and cool [9]. Boxes are generally placed on the ground with the opening facing west to minimize the influence of direct sunlight during the early part of the day. In wellshaded areas, the exact direction of the open end becomes less important $[2,7,9]$. It has been shown that female mosquitoes generally prefer larger and natural resting sites over smaller and artificial resting sites, respectively [10] and that in most cases the numbers of mosquitoes collected do not correlate with the results of host-seeking collections baited with humans [11]. This study investigated the ability of these boxes as outdoor devices for sampling host-seeking mosquitoes in urban Dar es Salaam where they had never been assessed before (Figure 1).

A new device for outdoor sampling adult mosquitoes called the Ifakara tent trap-B (ITT-B) has been developed and evaluated in both rural and urban Tanzania [8]. This trap operates passively all night long without skilled personnel and is designed to prevent exposure of the human volunteer that acts as bait by sleeping inside it. Field studies, in both rural Kilombero Valley and urban Dar es Salaam, have established that the new trap is efficacious in the sense that it has sufficient sensitivity to represent a viable alternative to HLC [8]. This study evaluated the effectiveness of the ITT-B and SRB relative to that of the HLC under field conditions of the Dar es Salaam's UMCP.
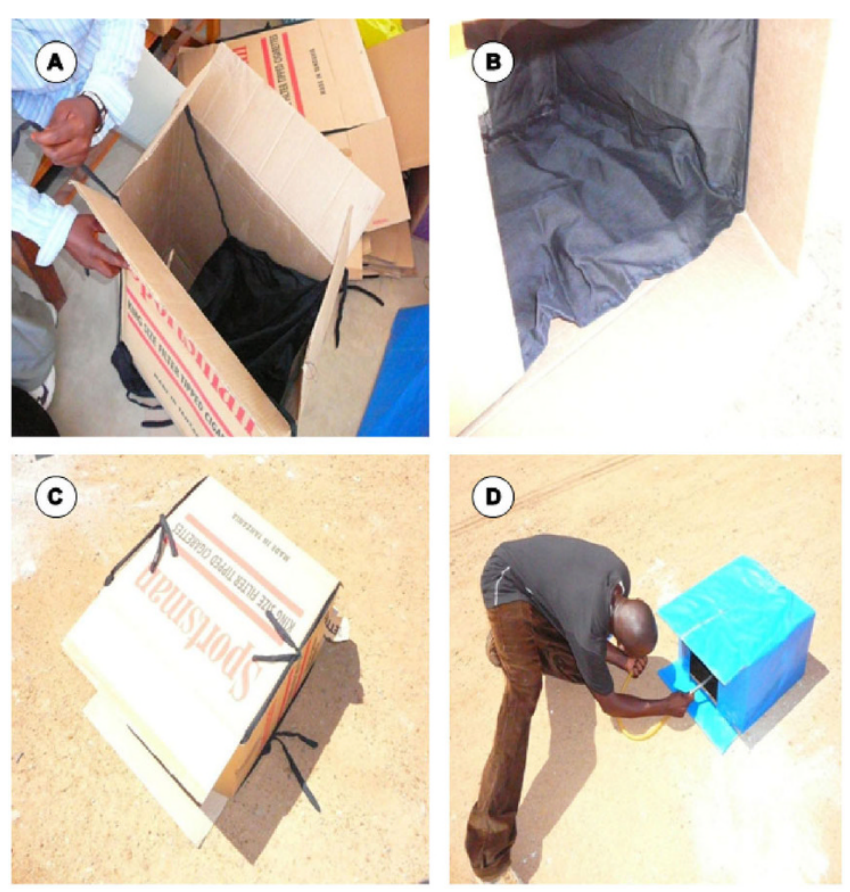

Figure I

Photographs of the standardized resting boxes (SRB) used in this study. Panels $A$ and $B$ illustrate how the boxes are made, panel $C$ demonstrates the way to install them, and panel $D$ demonstrates how to recover resting mosquitoes.

\section{Methods \\ Study site}

The study was carried out in Dares Salaam where a new Urban Malaria Control Programme has recently been reinitiated and a variety of associated studies on malaria vectors have been carried out [4-6,12-15]. The area experiences modest malaria transmission intensity with an entomologic inoculation rate (EIR) $[16,17]$ of approximately one infectious bite per person per year $[4,6]$. The main malaria vectors are members of the An. gambiae complex that predominantly feed out-doors [5].

Dar es Salaam is a coastal city in Tanzania (6'46' S Latitude and 39'14 E Longitude) with approximately 2.7 million inhabitants living in an administrative region which covers a total area of $1400 \mathrm{~km}^{2}$ [18]. Dar es Salaam has two rainy seasons: the short rains from late October to early December and the long rains from March to June. The climate is warm and tropical, with temperatures averaging $27^{\circ} \mathrm{C}\left(80^{\circ} \mathrm{F}\right)$ and rainfall varying from 750 to $1,400 \mathrm{~mm}$ per year. During the dry season temperatures often exceed $35^{\circ} \mathrm{C}$.

The UMCP covers 15 wards of urban Dar es Salaam, encompassing a surface area of $55 \mathrm{~km}^{2}$ with a total population of 609,514 people [4]. It operates primarily at the grassroots level through street health committees, using a 
community-based system originally developed by one of the three municipal councils (Ilala) that comprise the administrative region of Dar es Salaam. Presently, the program operates in five wards in each of the three municipalities (Ilala, Kinondoni and Temeke) as a communitybased pilot-scale program [14]. In 2004, the UMCP recruited and provided preliminary training to teams of Community Owned Resource Persons (CORPs) who performed weekly surveys of mosquito breeding habitats [12]. Operational larviciding in three selected wards with Bacillus thuringiensis var israelensis commenced in 2006 $[4,6]$. Currently, the UMCP implements four major activities in all the three municipalities: namely larval control, larval surveillance, adult mosquito surveillance and household parasitological surveys [4-6,14]. This study was based within the sampling frame and reporting system of the routine adult mosquito surveillance programme, which conducts monthly sampling of mosquitoes by HLC at 268 location distributed across the 15 wards of the UMCP $[5,6]$.

\section{Experimental design and selection of the sampling site for the three traps}

The study was carried out in 12 wards in the study area of the Urban Malaria Control Program. One neighbourhood (mtaa in Kiswahili) in each ward was randomly selected for this study. In each selected neighbourhood, four HLC sites already existed in four Ten Cell Units (TCUs) which were deliberately chosen to be well distributed across the neighbourhood and as close to potential breeding sites as possible. For each pre-existing HLC site, a nearby (100300 meters away) house was selected arbitrarily for both application of the ITT-B and the SRB. Therefore, in each neighbourhood, eight houses from different TCUs were used for the three sampling methods: four houses for the HLC and four houses for the ITT-B and the SRB, totaling 48 houses for the HLC and 48 for the combined ITT-B and SRB methods, respectively. Concomitant sampling with ITT-B and SRB began in the first enrolled wards in December 2007 and the last of the 12 wards had begun by March 2008. Data and mosquitoes collected up to end of June 2008 were included in this analysis, spanning a period of between seven and four months for each of the 12 wards.

\section{Field mosquito collection and processing}

Routine human landing catch was conducted outdoors once a week in each neighbourhood by one catcher working from 18.00 to 06.00 hours for a period of 45 minutes every hour, allowing the catcher to have a 15 minutes break. To minimize the possibility of data fabrication by the catchers, they were obliged to record the approximate number of each relevant mosquito taxon in their catches for each hour as they finished them. Moreover, spot checks were conducted inconsistently, unpredictably and at arbitrary times of the night by a team of 4 supervisors.
The mosquitoes caught were collected by the project vehicle the following morning and taken to the laboratory for further processing.

A protocol for sampling malaria vectors and other mosquitoes using the ITT-B and SRB was developed to enable community members to trap, record and submit samples of malaria vectors without any night-time supervision and only occasional contact with program staff. This protocol was used to evaluate the sensitivity of the ITT-B and the SRB relative to that of carefully controlled HLC as follows: Prior to the supply of materials for ITT-B and SRB experiments, demonstrations were provided to train the community-based staff on correct use of the two traps. The operators were supplied with all the necessary materials that allowed them to continuously collect mosquitoes for a period of one week while recording them on a form they were provided with. Mosquitoes trapped in the ITT-B were carefully aspirated using hand-held aspirators and placed into paper cups, once in the middle of each night $(00.00-$ $01.00)$ and then early in the morning the next day (05.0006.00). Operators were allowed to choose, at their own discretion, which nights of every week they slept in the traps and what time they entered and left the trap, under the condition that they recorded these dates and times. While still in paper cups, the mosquitoes were suffocated with a small ball of cotton wool soaked in petroleum ether. The dead mosquitoes were then transferred into smaller silica gel-filled containers for storage and preservation with a label indicating the ward, mtaa, site and day of collection.

Resting boxes were installed nearby (10-20 m) the ITT-B in each neighbourhood. The boxes were emptied between 06.00 and 08.00 in the morning of each working day using hand-held aspirators. Since experiments with ITT-B and SRB ran concurrently, suffocation, preservation and submission to the laboratory was accomplished in exactly the same way and at the same time as those from the ITTB.

\section{Laboratory processing and analysis}

All the mosquitoes collected in the field by HLC were taken to the laboratory and killed by suffocation with chloroform. For mosquitoes caught by ITT-B and SRB, this process was completed in the field by the trap operators who submitted their samples for identification and laboratory processing after a one week period of sampling. In the laboratory, all mosquitoes were identified morphologically using taxonomic keys [19] according to sex as males or females, morphologically as An. gambiae s.l., Anopheles funestus, Anopheles coustani, Cx. species, or Aedes species while the abdominal status was scored as gravid/ semi-gravid, fed or unfed for all the An. gambiae s.l. and for a manageable proportion of $C x$. species. All An. gambiae 
s.l. caught by the three trapping methods were subsequently desiccated over silica gel and kept at room temperature until they were further processed.

A wing or a leg of every An. gambiae s.l. mosquito caught was analyzed by PCR to identify its exact species within the An. gambiae complex [20]. An enzyme-linked immunosorbent assay (ELISA) using a monoclonalantibody that recognizes a repetitive epitope on the circumsporozoiteprotein of Plasmodium falciparum was used to assessmalaria sporozoite infection status in each individual $A n$. gambiae s.l. [21].

\section{Data handling and analysis}

All data handling and analysis was conducted with Microsoft Excel ${ }^{\circledR} 2007$ and SPSS ${ }^{\circledR} 15.0$. The only mosquito taxa considered for analysis were An. gambiae s.l. and Cx. species because these were the only ones for which sufficient numbers were collected throughout the study period.

To allow direct comparison with HLC conducted in the same area and in the same week, data was first aggregated by station and week, giving a total of 48 mean catches for matching station-week combinations over a period of 30 weeks. Prior to this analysis step, the numbers in each catch $(\mathrm{x})$ were normalized by transforming to $\log _{10}[\mathrm{x}+1]$ [3]. The relationship between catches by ITT-B or SRB and that of the HLC, in the same week and station, was initially assessed using simple Pearson's linear correlation method. Regression using generalized estimating equations was used to test for density dependence of the relative sampling efficiency of the ITT-B and SRB methods relative to the sum of the ITT-B and the HLC. On several occasions, the three traps recorded zero values for $A n$. gambiae s.l. mosquitoes even after aggregation by stationweek so no logical comparison could be made and these data were discarded. Since divisions by zero gives infinite values, data for several week-site observations were sorted by the sum of the catches for the traps (alternative plus the reference) and then aggregated by this sum with the mean of each of the two catches as the summary variables. The mean catch of the alternative collection methods divided by the mean catch of the reference method was treated as the dependent variable with a log link function and a gamma distribution for An. gambiae s.l. and a normal distribution for $C x$. species. The sum of the alternative and the reference methods was treated as a continuous independent variable in the model.

To test for consistent variations in species composition, sporozoite prevalence and abdominal condition of the mosquitoes sampled by the different traps, binary logistic regression in SPSS was used. Each outcome was treated as a binary variable with trap design as an independent categorical factor in the model. The results of abdominal status and sibling species identity were expressed as binary outcomes: fed (partially or fully-fed) versus non-fed (gravid or unfed) and An. gambiae s.s. versus An. arabiensis, respectively, as described previously [8]. Although sporozoite infection status was determined in the laboratory and the dependence of sporozoite prevalence upon trap type was tested for using a similar statistical approach, the number of mosquitoes caught was not sufficient to enable meaningful conclusions to be reached regarding this relatively rare fraction of the vector population.

\section{Ethical consideration and informed consent}

Informed consent was obtained from all the participants, namely the household owners and the mosquito catchers. Moreover, thick and thin blood smears were taken from all the participants whenever they complained of fever to examine the presence of malaria parasites. When found positive, they were treated with Coartem ${ }^{\circledR}$ (artemetherlumefantrine).

\section{Results}

\section{Overall performance of the three trapping method}

A total of 44,848 mosquitoes were collected during the entire study period of seven months. The composition of the sample was $98.9 \% C x$. species, $0.8 \%$ An. gambiae s.l., $0.2 \%$ Aedes species and $0.1 \%$ An. coustani. The ITT-B, HLC and SRB accounted for $59 \%, 30 \%$ and $11 \%$ of the total number of mosquitoes caught respectively. Over the entire sampling period, the SRB caught only 46 and 4,791 An. gambiae s.l. and $C x$. species, respectively. The total catches of $C x$. species and An. gambiae s.l. are outlined in further detail in Table S1 (Additional file 1).

There was a significant correlation between the mean weekly numbers of female An. gambiae s.l. caught by the ITT-B and the HLC for both Cx. species and An. gambiae s.l. in the 48 sampling sites (Table S2 (Additional file 2) and Figure 2). However, there was no correlation between the SRB and the HLC for An. gambiae s.l. even though a significant correlation existed for the $C x$. species. Both the ITT$\mathrm{B}$ and SRB showed no density dependence for the relative sampling efficiency of An. gambiae s.l. and Cx. species (Table S3 (Additional file 3) and Figure 3).

\section{Dependence of abdominal condition and species composition upon trapping method}

The distribution of abdominal conditions observed for $C x$. species and An. gambiae s.l. is presented in Table S4 (Additional file 4). An abdominal condition was determined for 12,776 Cx. species and 305 An. gambiae s.l No difference was observed between proportions of fed $A n$. gambiae s.l. captured by the ITT-B and the HLC. Furthermore, for $C x$. species, the ITT-B sampled a significantly higher number of fed mosquitoes than the HLC.

Of the 268 female An. gambiae s.l. analyzed by PCR for sibling species identification, an amplification success rate of 


\section{An. gambiae s.l.}

\section{Cx. species}
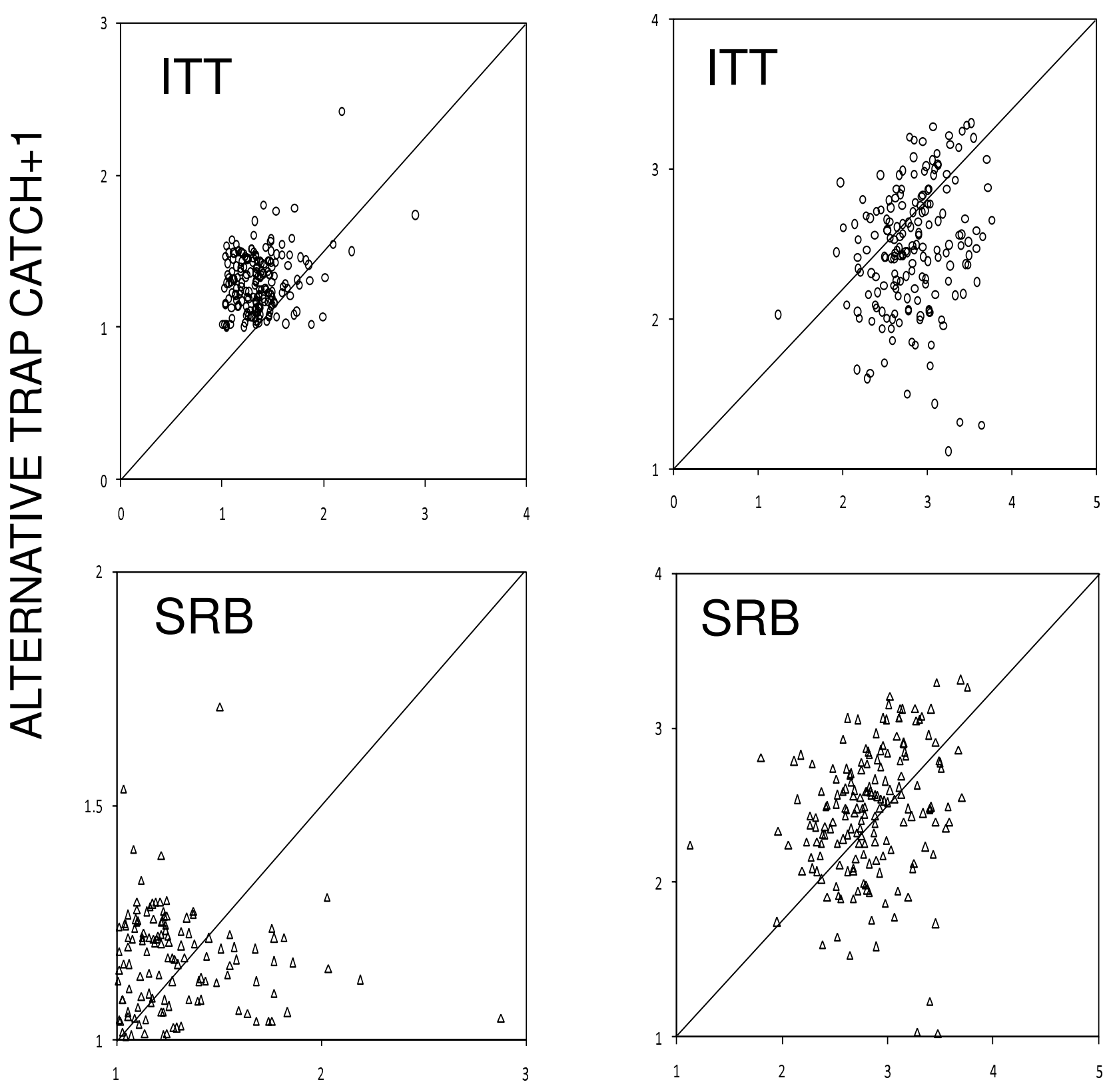

\section{HLC- REFERENCE TRAP CATCH +1}

Figure 2

Relationship between the log of the mean weekly numbers of female An. gambiae s.l. and Cx. species. It includes on overall 48 sampling stations over a period of 30 weeks. All values $(X$ or $Y$ ) are presented as $X+I+S$ or $Y+I+S$ where $S$ is a random number between 0 and 0.3 added to allow separation and visualization of otherwise identical data points. 


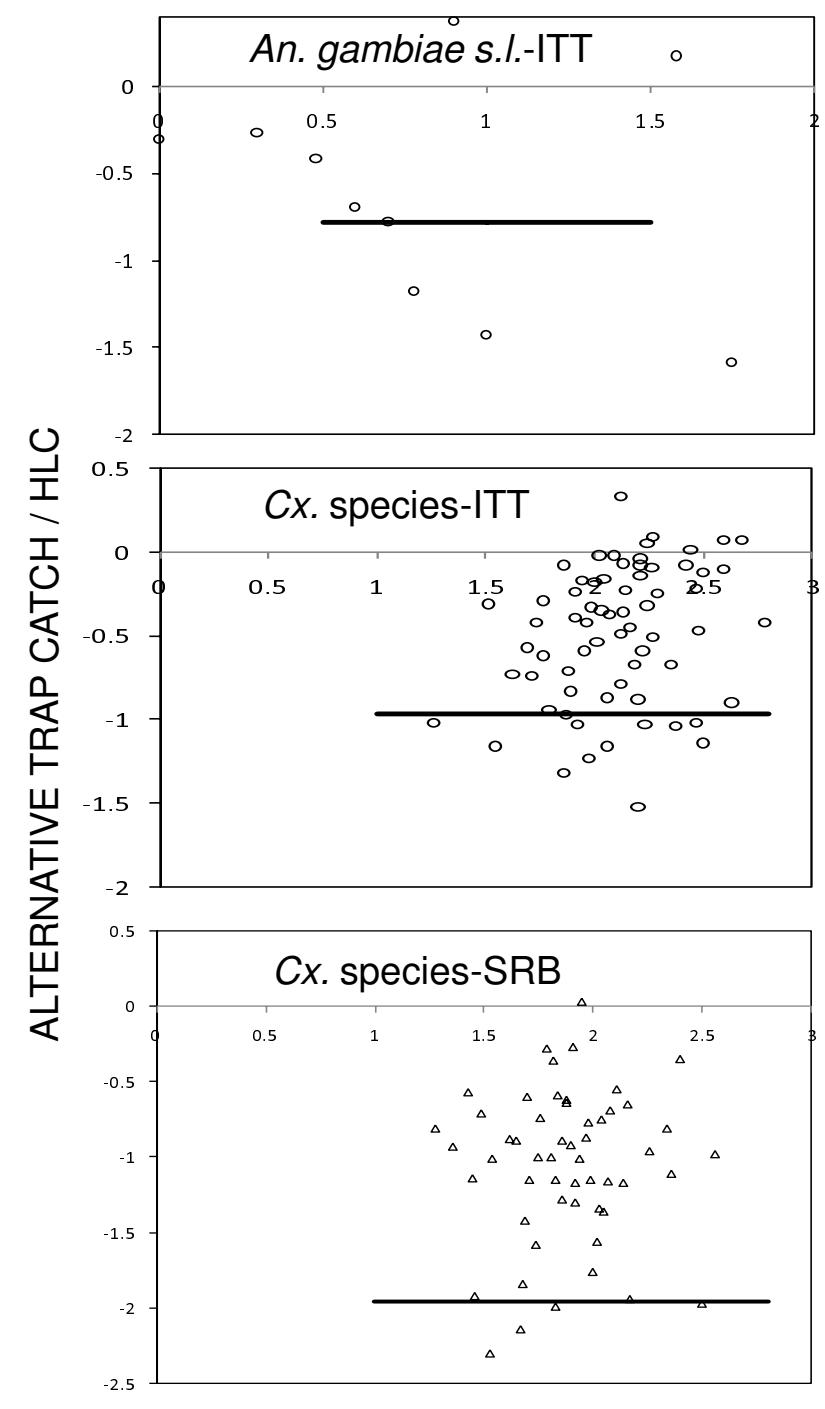

HLC+ALTERNATIVE TRAP CATCH

\begin{abstract}
Figure 3
Density dependence for the relative sampling efficiency of the ITT-B, HLC and SRB for An. gambiae s.I and $C x$. species. Each point on $X$ axis show the sum number of female An. gambiae s.l. and $C x$. species caught by the HLC and the alternative trap with several week-site observations. Solid lines depict the density-dependent sampling efficiency model.
\end{abstract}

$67.7 \%$ was obtained, leaving 87 specimens undetermined (Table S5) (Additional file 5). Of the successful amplifications, 83.3\% were An. gambiae s.s, 15.5\% Anopheles arabiensis with the remaining 1.2\% being Anopheles merus. Both An. gambiae s.s. and An. arabiensis were caught by all three sampling methods but notably the SRB yielded comparatively fewer An. arabiensis while only the HLC recorded An. merus (two specimens). Analysis using binary logistic regression showed no significant difference in proportion of An. gambiae s.s. versus An. arabiensis sampled by the three traps.

The sporozoite prevalence did not vary significantly between the mosquitoes sampled by the three trapping methods simply because the sample size was too small to make a meaningful analysis.

\section{Evaluation of the protocol in terms of cost-effectiveness}

Table S6 (additional file 6) summarizes the initial cost per week per sampling station, running costs per week per sampling station, total cost and the cost of sampling one mosquito caught for both the ITT-B and the HLC. In all cases, the HLC was more costly than the ITT-B. For example, it was found out that weekly sampling with the HLC (one night of collection) in one sampling station was roughly equivalent in cost to three weeks of sampling with the ITT-B (nine nights of collection) distributed across 3 sampling stations. Also, ITT-B was three and five times cheaper than the HLC per mosquito caught for An. gambiae s.l. and $C x$. species, respectively.

\section{Discussion and conclusion}

More An. gambiae s.l. were caught by the ITT-B than by the HLC or SRB. The ITT-B and SRB caught between 35\% and $15 \%$, respectively, of the number of An. gambiae s.l. caught per night by HLC. Note, however, that by applying the ITT-B for three nights in the same sampling site, its relative sensitivity per week matched the HLC for An. gambiae s.l. and exceeded it for $C x$. species. Interestingly, male mosquitoes of almost all the species sampled were found more frequently in the ITT-B than any other sampling method. It should be noted that male mosquitoes are just as useful an indicator of success or failure of a larval control programme even though they do not cause disease. Furthermore, male mosquitoes play an essential role in the life cycle of all mosquitoes and monitoring systems for genetic control strategies such as the release of sterile or genetically modified mosquitoes. The results of this study show that the ITT-B was the most efficient method for collecting An. gambiae s.l. and Cx. species per week because it was possible to conduct more intensive sampling with far less effort. SRB, normally considered as the method of choice for recovering resting mosquito populations in a variety of ecological settings, was insufficiently sensitive in urban Dar es Salaam.

Overall, the ITT-B was by far the most cost-effective sampling method. On the basis that the trap can handle more sampling nights than the HLC at a dramatically reduced cost, more sensitive, extensive, intensive and representative measurements of biting density can be determined over larger sampling areas. The fact that the ITT-B samples the vectors with minimal supervision while the HLC 
requires intense scrutiny and correspondingly substantial resources, are the primary reason for the difference in their overall costs. Whereas HLC involved daily use of a vehicle to distribute the sampling materials to the respective sampling sites, spot checks as well as picking the vectors the next day, the ITT-B involved none of these. The other major differential cost associated with the HLC was the diagnosis and treatment of the HLC catchers in case of any reported fever. By comparison, the ITT-B requires little or no maintenance so after the initial, expensive outlay of purchasing the traps themselves, it is remarkably affordable because these are very durable and the procedure does not require skillful personnel, intensive supervision or medical expense.

The failure of the ITT-B to reduce the proportion of blood fed mosquitoes suggests that exposure of the occupants does in fact occur, probably during the collection process which necessitates opening of the long zipper that bisects the protective panel. Subsequent follow up discussions with the operators revealed that indeed they do receive bites during the collection process. Therefore, an improved design will be required for routine use. One major disadvantage of the ITT-B often reported by the catchers was the fact that the trap was too heavy to be moved from one sampling station to the next by a single person. This problem was later solved by supplying the operators with bicycles. Also, occasionally the trap was reported to attract other insects but none of these were confirmed to be potential mosquito predators. Otherwise, the protocol was generally well accepted by the trap operators and appears to be easy enough for performance to be maintained with relatively modest incentives.

On the other hand, the SRB proved to be very impractical and on several occasions they were either soaked by rain or stolen. It also often proved difficult to retrieve the mosquitoes from the SRB. These problems, combined with their poor sensitivity and other sundry logistical matters appear to rule out the SRB as a candidate tool for routine mosquito sampling in the city of Dar es Salaam. The SRB have been evaluated previously in terms of efficacy and found to correlate poorly with the HLC [11] and this study reinforces that view. In earlier studies, it was found out that the proportion of adult mosquitoes resting in manmade shelters depended on the availability of alternative resting sites which varies according to location and changes seasonally [7] and in a recent study that female mosquitoes prefer larger resting sites over smaller ones [10]. Combining these considerations with the poor sensitivity observed, it appears that SRB are unlikely to provide a useful alternative to the HLC for sampling hostseeking malaria vectors in urban Dar es Salaam, particularly under operational conditions.
The correlation results obtained for ITT-B from this effectiveness trial were slightly different from those of efficacy trials by others $[3,8,22,23]$ (Table S2) (Additional file 2). For example, previous efficacy trials by Govella and others [8] recorded a much stronger correlation between ITT-B and HLC, than seen in this study. This is most probably because this study was carried out under conditions that involved minimal supervision compared to the intensely controlled efficacy trials. Another likely contributor to this weakened association is the fact that more sampling stations across a very heterogeneous environment were included in this study compared to the relatively few sampling stations for the efficacy trials. Nonetheless, the significant positive correlation between the HLC and the ITT$B$ (Additional file 2) suggest that this approach may be very useful in programmatic setting and provides a reasonably sensitive and accurate reflection of true mosquito biting densities.

Although the use of window traps installed in existing houses and emptied by resident community-based workers has been described as an effective tool for routine monitoring of indoor-residual spray programmes in southern Africa [24], no other effectiveness study of this kind has been reported for malaria vector trapping methods. The ITT-B not only represents an option for more accurate and representative measurement of human biting rate over a large sampling area, it is also practical and affordable to use in community-based sampling schemes. Nevertheless, it is recommended that the trap be evaluated in the longer term and on full programmatic scales until the effectiveness of this approach in fully representative conditions of routine practice is established. However, the largest remaining concern is probably the surprisingly high proportion of blood-fed mosquitoes caught, suggesting the design needs to be adapted to avoid human exposure during the empting process before it can be adopted as a routine mosquito-trapping tool.

\section{Competing interests}

The authors declare that they have no competing interests.

\section{Authors' contributions}

MS developed the protocol for mosquito sampling, supervised the field work, recorded and analyzed the data and drafted the manuscript. NJG designed the Ifakara tent trap-B design and assisted in interpretation of the results. $\mathrm{JM}$, SHK and PC supervised the field work and facilitated the implementation of the protocol. SBO assisted in data collection, data recording and implementation of the protocol. KK was involved in the planning and implementation of the protocol. WRM helped in drafting the study objectives and the manuscript. GFK conceived the study and oversaw the development of the experimental design, 
interpretation of the results, data analysis and drafting of the manuscript. All authors read and approved the final manuscript.

\section{Additional material}

\section{Additional file 1}

Summary of the totals, means and relative sensitivity of An. gambiae s.l. and Cx. species caught by the ITT-B, HLC and SRB. Summary of An. gambiae s.l. and Cx. species catches by the three traps.

Click here for file

[http://www.biomedcentral.com/content/supplementary/14752875-8-197-S1.pdf]

\section{Additional file 2}

Results from this study compared to other studies evaluating correlation between the HLC catches and alternative traps for female An. gambiae s.l. The data compares correlation between the catches of An. gambiae s.l. caught by the HLC and the alternative traps in this study and previous efficacy studies.

Click here for file

[http://www.biomedcentral.com/content/supplementary/14752875-8-197-S2.pdf]

\section{Additional file 3}

Regression analysis using generalized estimating equations (GEE) to determine density dependence relative sampling efficiency of the ITT$B$ and the SRB for An. gambiae s.l. and the Cx. species. Statistical analysis to indicate the sampling efficiency of the ITT-B and the HLC in terms of the vector density.

Click here for file

[http://www.biomedcentral.com/content/supplementary/14752875-8-197-S3.pdf]

\section{Additional file 4}

Abdominal condition scored by the three traps for An. gambiae s.l. and $\mathrm{Cx}$. species and the influence of each trap on the fed mosquitoes determined by binary logistic regression. The data represent statistical analysis of the abdominal status of the three traps.

Click here for file

[http://www.biomedcentral.com/content/supplementary/14752875-8-197-S4.pdf]

\section{Additional file 5}

Species composition of An. gambiae complex for the ITT-B, HLC and $S R B$ and the influence of each trap upon the proportion of An. gambiae s.s. sampled, as determined by binary logistic regression. The data presented is a summary of statistical analysis of the species composition of the three traps as determined by PCR.

Click here for file

[http://www.biomedcentral.com/content/supplementary/1475-

2875-8-197-S5.pdf]

\section{Additional file 6}

Comparative evaluation of cost effectiveness of the ITT-B and the HLC for weekly sampling and sampling a single An. gambiae s.l. and Cx. species. A summary of the cost of using the ITT-B and the HLC for sampling An. gambiae s.l. and Cx. species.

Click here for file

[http://www.biomedcentral.com/content/supplementary/14752875-8-197-S6.pdf]

\section{Acknowledgements}

We thank the people of Dar es Salaam for allowing us to use their premises for this research. We especially owe sincere gratitude to all those who volunteered to conduct the HLC and the ITT-B experiments. Both MS and SBO were supported by scholarships kindly provided by Valent Bioscience Corporation. This study was also supported by the Centers for Disease Control and Prevention and the United States Agency for International Development through US President's Malaria initiative, the Malaria Transmission Consortium (Bill \& Melinda Gates Foundation award number 45I I4) and a Research Career Development Fellowship (076806) provided to GFK by the Wellcome Trust.

\section{References}

I. De Savigny D, Binka F: Monitoring future impact on malaria burden in Sub saharan Africa. Am J Trop Med Hyg 2004, 71:224-231.

2. Mboera LEG: Sampling techniques for adult Afrotropical malaria vectors and their reliability in the estimation of entomolological innoculation rate. Tanzania Health Res Bull 2005, 7: I 17-124.

3. Lines JD, Curtis CF, Wilkes TJ, Njunwa KJ: Monitoring human-biting mosquitoes (Diptera: Culicidae) in Tanzania with lighttraps hung beside mosquito nets. Bull Entomol Res I991, 8I:77-84.

4. Fillinger U, Kannady K, William G, Vanek M, Dongus S, Nyika D, Geissbühler Y, Chaki P, Govella N, Mathenge E, Singer B, Mshinda H, Lindsay S, Tanner M, Mtasiwa D, de Castro M, Killeen G: A tool box for operational mosquito larval control: preliminary results and early lessons from the Urban Malaria Control Programme in Dar es Salaam, Tanzania. Malar J 2008, 7:20.

5. Geissbühler Y, Chaki P, Emidi B, Govella N, Shirima R, Mayagaya V, Mtasiwa D, Mshinda H, Fillinger U, Lindsay S, Kannady K, de Castro M, Tanner M, Killeen G: Interdependence of domestic malaria prevention measures and mosquito-human interactions in urban Dar es Salaam, Tanzania. Malar 」 2007, 6: 126.

6. Geissbühler Y, Kannady K, Chaki PP, Emidi B, Govella NJ, Mayagaya V, Kiama M, Mtasiwa D, Mshinda H, Lindsay SW, Tanner M, Fillinger $U$, de Castro MC, Killeen GF: Microbial larvicide application by a large-scale, community-based program reduces malaria infection prevalence in urban Dar Es Salaam, Tanzania. PLoS ONE 2009, 4:e5I07.

7. Service MW: A critical review of procedures for sampling populations of adult mosquitoes. Bull Entomol Res 1977, 67:343-382.

8. Govella N, Chaki P, Geissbuehler Y, Kannady K, Okumu F, Charlwood J, Anderson R, Killeen G: A new tent trap for sampling exophagic and endophagic members of the Anopheles gambiae complex. Malar J 2009, 8:157.

9. Wayne JC: Resting boxes as mosquito surveillance tools. Proceedings of the Eighty-Second Annual Meeting of the New Jersey Mosquito Control Association 1989:53-57.

10. Burkett DN, Eubanks DM, Unnasch RT: Preference of female mosquitoes for natural and artificial resting sites. J Am Mosq Control Assoc 2008, 24:228.

11. Kay BH: Collection of resting adult mosquitoes at Kowanyama, Northern Queensland and Charleveville, South West Queensland. Aust J Entomol 1982, 22:19-24.

12. Vanek MJ, Shoo B, Mtasiwa D, Kiama GM, Fillinger U, Kannady K, Tanner M, Killeen GF: Community-based surveillance of malaria vector larval habitats: A baseline study in urban Dar es Salaam, Tanzania. BMC Public Health 2006, 6: 154

13. Sattler MA, Mtasiwa D, Kiama M, Premji Z, Tanner M, Killeen GF, Lengeler C: Habitat characterization and spatial distribution of Anopheles sp. mosquito larvae in Dar es Salaam (Tanzania) during an extended dry period. Malar J 2005, 4:4.

14. Mukabana WR, Kannady K, Kiama GM, ljumba J, Mathenge EM, Kiche I, Nkwengulila G, Mboera LEG, Mtasiwa D, Yamagata Y, van Schayk I, Knols BG], Lindsay SW, Caldas de Castro M, Mshinda H, Tanner M, Fillinger U, Killeen GF: Ecologists can enable communities to implement malaria vector control in Africa. Malar J 2006, 5:9.

15. Castro MC, Yamagata Y, Mtasiwa D, Tanner M, Utzinger J, Keiser J, Singer $\mathrm{BH}$ : Integrated urban malaria control: a case study in Dar es Salaam, Tanzania. Am J Trop Med Hyg 2004, 7I: 103-I I7. 
16. Beier JC, Killeen GF, Githure J: Short report: Entomologic inoculation rates and Plasmodium falciparum malaria prevalence in Africa. Am J Trop Med Hyg 1999, 61:109-113.

17. Smith DL, McKenzie FE, Snow RW, Hay SI: Revisiting the basic reproductive number for malaria and its implications for malaria control. PLOS Biol 2007, 5:42.

18. The 2002 population and housing census general report. Dares Salaam: Government of Tanzania. National Bureau of Statistics 2003.

19. Gillies MT, Coetzee M: A supplement to the Anophelinae of Africa South of the Sahara (Afrotropical region). Johannesburg 1987.

20. Scott JA, Brogdon WG, Collins FH: Identification of single specimens of the Anopheles gambiae complex by the polymerase chain reaction. Am J Trop Med Hyg 1993, 49:520-529.

2I. Burkot TR, Williams JL, Schneider I: Identification of Plasmodium falciparum-infected mosquitoes by a double antibody enzyme-linked immunosorbent assay. Am J Trop Med Hyg 1984, 33:783-788.

22. Magbity EB, Lines JD, Marbiah MT, David K, Peterson E: How reliable are light traps in estimating biting rates of adult Anopheles gambiae s.l. (Diptera: Culicidae) in the presence of treated bed nets? Bull Entomol Res 2002, 92:7I-76.

23. Mbogo CN, Glass GE, Forster D, Kabiru EW, Githure JI, Ouma JH, Beier JC: Evaluation of light traps for sampling anopheline mosquitoes in Kilifi, Kenya. J Am Mosq Control Assoc 1993, 9:260-263.

24. Sharp BL, Kleinschmidt I, Streat E, Maharaj R, Barnes KI, Durrheim DN, Ridl FC, Morris N, Seocharan I, Kunene S, JJ LAG, Mthembu JD, Maartens F, Martin CL, Barreto A: Seven years of regional malaria control collaboration - Mozambique, South Africa, and Swaziland. Am J Trop Med Hyg 2007, 76:42-47.

Publish with Bio Med Central and every scientist can read your work free of charge

"BioMed Central will be the most significant development for disseminating the results of biomedical research in our lifetime. "

Sir Paul Nurse, Cancer Research UK

Your research papers will be:

- available free of charge to the entire biomedical community

- peer reviewed and published immediately upon acceptance

- cited in PubMed and archived on PubMed Central

- yours - you keep the copyright

Submit your manuscript here:

http://www.biomedcentral.com/info/publishing_adv.asp
BioMedcentral 